\title{
Stature Estimation of the Igbos Using Cephalo-Facial Anthropometry
}

\author{
Jervas $\mathrm{E}^{1^{*}}$, Anibeze $\mathrm{CIP}^{2}$, Uloneme $\mathrm{GC}^{3}$ and Anyanwu $\mathrm{GE}^{4}$ \\ ${ }^{1}$ Department of Prosthetics and Orthotics School of Health Technology, Federal University of Technology, Owerri, Nigeria \\ ${ }^{2}$ Department of Anatomy, College of Medicine, Abia State University, Uturu, Nigeria \\ ${ }^{3}$ Department of Anatomy, College of Medicine, Imo State University Owerri, Nigeria \\ ${ }^{4}$ Department of Anatomy, College of Medicine, University of Nigeria, Enugu Campus, Nigeria
}

"Corresponding author: Ekezie Jervas, Department of Prosthetics and Orthotics, School of Health Technology, Federal University of Technology, P.M.B 1526, Owerri, Nigeria, Tel: +234 806 5430037; E-mail: ekeziejervas@gmail.com

Rec date: May 11, 2015 Acc date: July 25, 2015 Pub date: July 30, 2015

Copyright: (c) 2015 Jervas E, et al. This is an open-access article distributed under the terms of the Creative Commons Attribution License, which permits unrestricted use, distribution, and reproduction in any medium, provided the original author and source are credited.

\begin{abstract}
Estimation of stature is one of the biological profiles used in the identification of individuals in cases of mass disasters, plane crash, fatal auto crash, bombing, homicides, and in crime investigations. It is an important tool in Medico legal practice.

In the present study an attempt has been made to derive regression formulae to determine stature from cephalofacial dimensions. Informed consent was obtained and measurements were taken following standard protocols. The prediction function was derived through linear regression and multiple regressions for each of the measurement with stature, for the general population and for males and females separately. Cephalo-facial dimensions of 211 subjects females $n=123$ and males $n=88$ with mean age $23.58 \pm 4.95$ belonging to the Igbo population of Nigeria were studied. Eleven cephalo-facial measurements including stature were taken. The mean stature of both genders put together was $167.55 \pm 9.10 \mathrm{~cm}$ while that of females and males were $163.17 \pm 7.64 \mathrm{~cm}$ and $173.66 \pm 7.30 \mathrm{~cm}$ respectively. Males were significantly taller than the females $(p<0.0001)$. Accuracy of determination 'R' of stature from Cephalo-facial dimensions was highest for IOB and EBB in both genders put together; it was also highest using IOB in the females while MFH and MHB yielded that of the males.
\end{abstract}

Keywords: Head; Face; Dimensions; Igbos; Cephalo-facial dimensions

\section{Introduction}

Cephalo-facial anthropometry has been employed by many authors to estimate stature of the human body Patil and Mody [1], Krishaan (2008), Rayan and Bidmos (2007), Sahni et al., Chiba and Terazawa $[2,3]$, because forensic investigators may be confronted with highly decomposed and mutilated dead bodies with fragmentary remains involving the head (cephalo-facial region). Ileyperuma (2010), investigated the relationship between stature and cranial dimensions in Sri Lanka, and proposed a gender and age specific linear regression model between the cranial dimensions and the height of an individual. There are also reports that odontery and anthropometric data of the skull provide reliable method of estimation of height from skeletal remains in the forensic setup, Kalia et al. [1-5].

With the emergence of the importance of stature estimation from body parts as a pointer to determine, the age, sex, stature and race (the "Big Four" of Forensic Anthropology) to which a person belong, and in the dictation of criminals, the present study is designed to estimate stature of the Igbos using cephalo-facial anthropometry.

The Igbo, Pronounced ee-bo, are a group of distinguished people who live in the Southeastern Nigeria.

They live in villages, towns and cities scattered over the Eastern part of Nigeria, South of the River Benue and East of River Niger, which is in the rain forest belt of the country [6].
The Igbos has an area of about 15,800 square mile (about 41,000 square kilometers) between latitude $5^{\circ}$ and $7^{\circ}$ north and between $6^{\circ}$ and $8^{\circ}$ east of the Greenwich Meridian. They are bounded to the east by the lands of the Ibibio and the Cross River, to the south by the Ijo speaking people, to the west by the Edo ethnic group and to the north by the Igala and the Idoma speaking people $[7,8]$.

\section{Estimation of Stature from Cephalo-Facial Measurements}

Nine cephalo-facial measurements (serial numbers1-9 below) of each subject along with their stature $(\mathrm{Y})$ were measured following the conventional methods of (Weiner and Lourie 1981), Lohman et al. (1988), Hall et al. (2003) Krishan and Kumar (2007) plus three other cephalo-facial measurements taken from the ear and mouth [9-11].

Cephalo-facial measurements were taken with the help of a sliding caliper and measuring tape to the nearest $0.1 \mathrm{~cm}$. Only subjects without any physical body abnormality were included in the study.

- Stature (S): It measures the greatest distance from the plane where the subjects sands to the vertex (V) on the head using Anthropometer.

- Morphological Facial Height (MFH): it measures the straight distance from the nasion $(\mathrm{N})$ to the gnathion $(\mathrm{Gn})$.

- Physiogonomic Facial Height (PFH): it measures the straight distance between trichion (Tr) and gnathion (Gn).

- Maximum head circumference (MHC): This measures the maximum circumference of the head from the glabella area 
(usually horizontal just above the eyebrow ridges) to the area near the top of the occipital bone (opisthocranion).

- Maximum Head length (MHL): measures the straight distance between glabella (the most prominent point on the frontal bone above the root of the nose, between the eyebrows) and the opisthocranion (the most prominent portion of the occiput, close to the midline on the posterior rim of the foramen magnum).

- Maximum Head breath (MHB): measures the distance between the most lateral points of the parietal bone. It is also called maximum biparietal diameter.

- Bigonial diameter (BD): it is the maximum breadth of the lower jaw between two gonion points of the mandible. It represents the most posterior, inferior and laterally situated point on the external angles of the mandible.

- External Biocular Breadth (EBB): it measures the straight distance between ectocathion $(\mathrm{Ec})$ i.e., outer corners of the eye. It would be measured by using sliding caliper.

- Inter- Ocular Breadth (IOB): it measures the straight distance between endo-canthion (En).

- Ear height (EH): The straight distance between the most superior part of the auricle and the most inferior part of the lobule (ear lobe).

- Ear width (EW): The straight distance between the middle part of the helix and the middle attachment of the tragus to the skin of the face.

- Distance between the ear and the angle of the mouth (DBE\&AM): This measures the distance between the inferior attachment of the ear and the angle of the mouth in $\mathrm{cm}$.

\section{Data Presentation and Analysis}

The data analysis in this present study was carried out using statistical package for social sciences (SPSS 17.0 software).

The Minimum, Maximum, Mean and Standard deviations of the variables are presented.

Paired samples test was performed to check for statistical significant difference between the male's and female's cephalo-facial variables.

Pearson correlation was then applied to test the relationship between stature and cephalo-facial dimensions and the results are presented for the general population (both genders together), males and females.

The prediction function was derived through linear regression for each of the measurement with stature for both genders together, males and females separately. The presentation also provides the values of Constant, Regression coefficient, Percentage variation explained $\left(\mathrm{R}^{2}\right)$ and Significance of regression coefficient. The multiple linear regression models with the explanatory variables or repressor's cephalo-facial dimensions were proposed as a statistical model to explain the total variation (Dawnson and Trapp, 2004). The generated regression formula is such that stature $(y)=a \quad$ constant $)+b 1$ (regression coefficient for the first variable) $x_{1}$ (first variable) $+b_{2}$ (regression coefficient for the second variable) $\mathrm{x}_{2}$ (second variable) + $\ldots \ldots b_{n}$ (regression coefficient for the $n$th variable) $x_{n}$ ( $n$th variable), with this one can calculate stature from cephalo-facial dimensions.

\section{Result}

The total sample $(\mathrm{N}=211)$ for cephalo-facial anthropometry had a mean age of $23.58 \pm 4.95$ years, a mean stature of $167.55 \pm 9.10 \mathrm{~cm}$. The mean, standard deviation, minimum and maximum value of age, stature $(\mathrm{Y})$, and cephalo-facial variables of both genders put together, females and males are shown; males had higher values than the females.

In the paired samples test between females and males for the cephalo-facial variables, only bigonal diameter (BD) and morphological facial height (MFH) had significant values $(\mathrm{p}=0.028$, and $\mathrm{p}=0.046)$ at $95 \% \mathrm{CI}$ respectively. This shows that the mean BD and MFH for the males were significantly larger than that of the females.

The Pearson Correlation coefficients between stature and cephalofacial variables for both genders put together, females and males is shown. In both genders put together the External Biocular Breadth (EBB) and Inter-Ocular Breadth (IOB) were positively correlated with height $(\mathrm{P}<0.05 ; \mathrm{r}=0.150$ and $\mathrm{r}=0.181$, respectively).

The linear regression equations derived for the cephalo-facial measurement with stature for both gender indicated that the constant for EBB and IOB was 162.26 and 159.63 respectively. The regression coefficients (0.57 and 2.32) were significant indicating that they are contributing for the prediction of stature. The variation explained $\left(\mathrm{R}_{2}\right.$ $x$ 100) showed that EBB contributes $2.2 \%$ to stature while IOB contributes $3.3 \%$. The multiple regression equations derived for the cephalo-facial measurement with stature for both gender indicated that the constant for EBB and IBB was 154.828. The regression coefficients were also significant indicating that they are contributing for the prediction of stature. The variation explained $\left(R_{2} \times 100\right)$ showed that multiple regressions for both EBB and IOB contributed $5.3 \%$ to stature. The best prediction power was observed in EBB and IOB combined.

The linear regression equations derived for the cephalo-facial measurement with stature for the females indicated that the constant for MFH and IBB was 181.845 and 148.186 respectively. The regression coefficients $(-1.327$ and 4.541$)$ were significant indicating that they are contributing for the prediction of stature. The variation explained $\left(\mathrm{R}_{2} \times 100\right)$ showed that MFH contributes $5.3 \%$ to stature while IOB contributes $5.8 \%$.

The linear regression equations derived for the cephalo-facial measurement with stature for the males indicated that the constant for MHB and MFH was 152.93 and 158.53 respectively. The regression coefficients (0.93 and 1.02) were significant indicating that they are contributing for the prediction of stature. The variation explained $\left(\mathrm{R}_{2}\right.$ $x$ 100) showed that MHB contributes $8.1 \%$ to stature while MFH contributes $4.7 \%$. The multiple regression equations derived for the cephalo-facial measurement with stature for males indicated that the constant for MHB and MFH was 137.947. The regression coefficients (0.948 and 1.017) were highly significant indicating that they are contributing for the prediction of stature. The variation explained $\left(\mathrm{R}_{2}\right.$ $x$ 100) showed that the multiple regression for both MHB and MFH contribute $12.8 \%$ to stature.

The mean predicted (estimated) value of stature through the regression function was similar to the mean observed (actual) value. This is because the regression equations were calculated from measures of central tendency; however the minimum and maximum value indicated that there were differences in the predicted and observed value. 
Regression equation, for estimation of stature from cephalo-facial measurements in both genders put together, females and males. There are separate equations for positively correlated cephalo-facial measurement which can help in estimation of stature from individual part of the head or face. The regression equations have been calculated by regression analysis, the value of constants 'a' and ' $b$ ' are calculated. ' $a$ ' is the regression coefficient of the dependant variable, i.e. stature, and ' $b$ ' is the regression coefficient of the independent variable, i.e. any of the cephalo-facial dimensions. The regression formulae were constructed from cephalo-facial measurement by substituting the appropriate values of constants ' $a$ ' and ' $b$ ' in the standard equation of regression line. A multiple regression analysis was also performed with two cephalo-facial parameters (i.e. EBB and IOB) put together for both genders put together. A multiple regression analysis was also performed with two cephalo-facial parameters (i.e. MHB and MFH) put together for the males. Presented is the standard error of estimate (SEE) and multiple regression coefficients (R). SEE tends to predict the deviation of estimated stature from the actual stature i.e. refers the error that may arise from estimating stature Ozaslan et al. (2003). A low value denotes greater reliability of prediction from a particular measurement while a higher value shows less reliability of prediction. $\mathrm{R}$ (the multiple correlation coefficient value) is one of the measures used for model adequacy. $\mathrm{R}$ is defined as the correlation between the observed values and the fitted values for the dependent variable (Dawnson and Trapp 2004).

\section{Discussion}

Eleven cephalo-facial measurements including stature of the subjects were taken. The prediction function was derived through linear regression and multiple regressions for each of the cephalofacial measurement with stature, for the general population and for males and females separately.

In this study, the mean stature and age for the general population is $167.55 \pm 9.00 \mathrm{~cm}$ and $23.58 \pm 4.95$ yrs respectively. While the minimum and maximum stature is $149.00 \mathrm{~cm}$ and $190.00 \mathrm{~cm}$ respectively. The mean stature and age for the female and male subjects are $163.17 \pm 7.64 \mathrm{~cm}, 23.74 \pm 5.36$ yrs. and $173.66 \pm 7.30 \mathrm{~cm}$ and $23.35 \pm 4.34$ yrs respectively.

In sexing the cephalo-facial parameters, only the bigonal diameter and morphological facial height showed significant difference $(\mathrm{P}=0.028, \mathrm{P}=0.046)$ respectively. These values were higher in the males than the females.

The findings of the present study indicate that not all the cephalofacial dimensions are positively and significantly correlated with stature. In this study only EBB and IOB were significantly correlated with stature $(\mathrm{P}<0.05)$ in the general population. In the females $\mathrm{MFH}$ and IOB were significantly correlated with stature while in the males only $\mathrm{MHB}$ and $\mathrm{MFH}$ indicated significant correlated with stature $(\mathrm{P}<0.05)$. The linear regression analysis showed that IOB contributed $3.3 \%$ with 8.98 Standard error of estimate (SEE) much to stature than EBB and would be more appropriate to be used in stature estimation for the population. However the multiple regressions constructed had a SEE of 8.91 and contributes $5.3 \%$ to stature and would be more ideal to be use in the general population than the linear regression generated. For both the female and male subjects the multiple regressions constructed contributed $9.1 \%$ and $12.8 \%$ respectively to the variation in stature than the linear regressions.
The SEE for the cephalo-facial measurement ranges between \pm 6.95 and $\pm 9.03 \mathrm{~cm}$. The highest value of SEE is exhibited by EBB $( \pm 9.03)$ in the general population using linear regression analysis. The least value of SEE is exhibited by MHB and MFH, $( \pm 6.95 \mathrm{~cm})$ of the females by multiple regressions analysis.

The result of this work can be compared with some available studies on different populations. Patil and Mody (2005), in their lateral cephalometric study showed somewhat higher standard errors for most of the parameters except head length that displayed high degree of reliability $(\mathrm{SE}=3.71)$ [1].

Krishan (2008) estimated stature from five cephalofacial measurements and noted a SEE of \pm 5.820 for MFH. In our study the SEE for MFH was $\pm 7.34 \mathrm{~cm}$ in males but BD did not yield any positive correlation with stature and could not be used to estimate stature in the population under study (Igbo). Krishan and Kumar (2007) were able to estimate stature from sixteen cephalo-facial measurements in a sample of Koli male adolescent of North India and reported a SEE of 4.41-7.21. In our own study eleven cephalo-facial measurements were taken but we were able to regress stature on $\mathrm{EBB}(\mathrm{SEE}= \pm 9.03)$, IOB $(\mathrm{SEE}= \pm 8.98)$, in the general population; in the females, stature was regress on $\mathrm{MFH}(\mathrm{SEE}= \pm 7.60)$, IOB $(\mathrm{SEE}= \pm 7.64)$ while in males stature was estimated using MHB (SEE $= \pm 7.09)$ and $\mathrm{MFH}(\mathrm{SEE}= \pm 7.23)$ only.

Maximum head circumference did not yield any positive correlation with stature in this study and could not be used to estimate stature in the Igbos. However Krishan (2008) estimated stature using horizontal circumference of the head which he indicated to be the best parameter to estimate stature when compared with other cephalofacial parameters.

Many of these studies (Patil and Mody 2005), (Krishan and Kumar 2007), (Ryan and Bidmos 2007), Krishaan (2008), estimated the stature using linear regression. Our study made attempt to estimate stature from cephalo-facial variables using multiple regressions. The multiple regressions analysis presented lower value of SEE as 8.91 when EBB and IOB is combined in the general population; $\mathrm{MFH}+\mathrm{IOB}=7.58$ in the females, $\mathrm{HB}+\mathrm{MFH}=6.95$ in the males when compared with the values obtained using linear regressions in the present study $[1,2]$.

\section{Conclusion}

Although some of the SEE obtained (6.95-7.58) in this study is in line with the values gotten in the other studies state above but the slight high value of SEE in some of our parameters may be due to genetic, environmental and nutritional factors. However this study has proved that stature could be estimate in the Igbos using some cephalofacial measurements (MHB, EBB, IOB and $\mathrm{MFH}$ ). The multiple regressions generated performed better than the simple regressions due to the value of $\mathrm{R}$ and SEE obtained.

\section{Acknowledgement}

The authors wish to express their sincere thanks to the subjects who participated in this study.

\section{References}

1. Patil KR, Mody RN (2005) Determination of sex by discriminant function analysis and stature by regression analysis: a lateral cephalometric study. Forensic Sci Int 147: 175-180.

2. Krishan K (2008) Estimation of stature from cephalo-facial anthropometry in north Indian population. Forensic Sci Int 181: 52. 
Citation: Jervas E, Anibeze CIP, Uloneme GC, Anyanwu GE (2015) Stature Estimation of the Igbos Using Cephalo-Facial Anthropometry. J Forensic Res 6: 1000295. doi:10.4172/2157-7145.1000295

Page 4 of 4

3. Chiba M, Terazawa K (1998) Estimation of stature from somatometry of skull. Forensic Sci Int 97: 87-92.

4. Ilayperuma I (2010) On the prediction of person stature from cranial dimensions. International Journal of Morphology 28: 1135-1140.

5. Kalia S, Shetty SK, Patil K, Mahima VG (2008) Stature estimation using odontometry and skull anthropometry. Indian J Dent Res 19: 150-154.

6. Buchanan KM, Pugh JC (1955) Land and people in Nigeria, London 58.

7. Nzimiro IK (1972) Studies in Igbo Political systems (Chieftaincy and Politics in four Niger States), London: Frank Cass 3.
8. Onwuka (2002) Nigeria Peoples and Cultures: Historical and SocioStructural perspectives. Echrisi and Company Nigeria 23.

9. Weiner JS, Lourie JA (1981) Human Biology- A guide to field methods, Blackwell scientific publication, Oxford and Edinburg.

10. Lohman T, Roche A, Martorell R (1988) Anthropometric Standardization Reference Manual. Library of Congress 3-26.

11. Hall JG, Froster-Iskenius UG, Allision JE (2003) Hand book of Normal Physical Measurements, Oxford University Press, New York, Tronto. 